Published in final edited form as:

J Artif Organs. 2017 December ; 20(4): 381-385. doi:10.1007/s10047-017-0958-5.

\title{
Generating Pulsatility by Pump Speed Modulation with Continuous-Flow Total Artificial Heart in Awake Calves
}

\author{
Kiyotaka Fukamachi ${ }^{1}$, Jamshid H. Karimov ${ }^{1}$, Gengo Sunagawa ${ }^{1}$, David J. Horvath ${ }^{1,2,{ }^{*} \text {, }}$ \\ Nicole Byram ${ }^{1}$, Barry D. Kuban ${ }^{3}$, Raymond Dessoffy ${ }^{1}$, Shiva Sale ${ }^{4}$, Leonard A. R. \\ Golding $^{1, *}$, and Nader Moazami ${ }^{5}$ \\ ${ }^{1}$ Department of Biomedical Engineering, Lerner Research Institute, Cleveland Clinic, Cleveland, \\ Ohio, USA
}

${ }^{2} \mathrm{R} 1$ Engineering, Cleveland, Ohio, USA

${ }^{3}$ Electronics Core, Lerner Research Institute, Cleveland Clinic, Cleveland, Ohio, USA

${ }^{4}$ Department of Cardiothoracic Anesthesiology, Anesthesiology Institute, Cleveland Clinic, Cleveland, Ohio, USA

${ }^{5}$ Department of Thoracic and Cardiovascular Surgery, Kaufman Center for Heart Failure, Heart and Vascular Institute, Cleveland Clinic, Cleveland, Ohio, USA

\begin{abstract}
The purpose of this study was to evaluate the effects of sinusoidal pump speed modulation of the Cleveland Clinic continuous-flow total artificial heart (CFTAH) on hemodynamics and pump flow in an awake chronic calf model. The sinusoidal pump speed modulations, performed on the day of elective sacrifice, were set at $\pm 15 \%$ and $\pm 25 \%$ of mean pump speed at $80 \mathrm{bpm}$ in four awake calves with a CFTAH. The systemic and pulmonary arterial pulse pressures increased to $12.0 \mathrm{~mm}$ $\mathrm{Hg}$ and $12.3 \mathrm{~mm} \mathrm{Hg}( \pm 15 \%$ modulation) and to $15.9 \mathrm{~mm} \mathrm{Hg}$ and $15.7 \mathrm{~mm} \mathrm{Hg}( \pm 25 \%$ modulation), respectively. The pulsatility index and surplus hemodynamic energy significantly increased to 1.05 and $1,346 \mathrm{ergs} / \mathrm{cm}$ at $\pm 15 \%$ speed modulation and to 1.51 and $3,381 \mathrm{ergs} / \mathrm{cm}$ at $\pm 25 \%$ speed modulation. This study showed that it is feasible to generate pressure pulsatility with pump speed modulation; the platform is suitable for evaluating the physiologic impact of pulsatility and allows determination of the best speed modulations in term of magnitude, frequency, and profiles.
\end{abstract}

\footnotetext{
Corresponding Author: Kiyotaka Fukamachi, MD, PhD, Director, Cardiovascular Dynamics Laboratory, Department of Biomedical Engineering/ND20, Cleveland Clinic, 9500 Euclid Avenue, Cleveland, OH 44195, Tel: 216-445-9344; Fax: 216-444-9198;

fukamak@ccf.org. Second Corresponding Author: Jamshid H. Karimov, MD, PhD, Department of Biomedical Engineering/ND20, Cleveland Clinic, 9500 Euclid Avenue, Cleveland, OH 44195, Tel: 216-445-3255; Fax: 216-444-9198; karimoj@ ccf.org. *Note: Coauthors LARG and DJH of the Department of Biomedical Engineering recently retired from the department.

Field of Research: Artificial Heart (basic)

Presented at the 36th Annual Meeting of International Society for Heart and Lung Transplantation, April 27-30, 2016, Washington, DC, USA

Conflicts of Interest

David Horvath, Barry Kuban and Leonard A.R. Golding are inventors of the continuous-flow total artificial heart. The technology was licensed to Cleveland Heart, Inc., a Cleveland Clinic spin-off company. None of the other authors have a financial interest or other potential conflict of interest related to subject matter or materials mentioned in the manuscript.
} 


\section{Keywords}

Heart-assist devices; Hemodynamics; Implants; experimental; Pulsatile flow; Waveform

\section{Introduction}

Over the past several years, continuous-flow (CF) left ventricular assist devices (LVADs), which are either axial or centrifugal in design, have replaced volume-displacement pulsatileflow pumps because of their simplicity, mechanical reliability, durability, small size, patient and caregiver acceptability, and improved outcomes. However, in patients with a CFLVAD, there have been increasing reports of the incidence of multiple complications, such as aortic valve insufficiency, gastrointestinal bleeding, pump thrombosis and hemolysis, cerebrovascular accident, renal dysfunction, and hypertension. Some of these could be related to reduced pulsatility of the arterial pressure [1-4].

There has been significant interest in evaluating the impact of generating pulsatile flow with continuous-flow pumps using speed modulation. The Cleveland Clinic continuous-flow total artificial heart (CFTAH) offers a unique platform because its operation can range from completely nonpulsatile flow at constant speed to pulsatile flow during speed modulations. The purpose of this study was to evaluate effects of sinusoidal pump speed modulation on hemodynamics and pump flow in an awake chronic calf model.

\section{Materials and Methods}

\section{Pump Description}

The CFTAH delivers blood flow to both the systemic and pulmonary circulation from a single pump assembly (one motor with one rotating assembly supported on a hydrodynamic bearing) [5,6]. Impellers supporting the left and right circulation are mounted on the two ends of the single rotating assembly, which is free to move axially, allowing the left and right hydraulic environments to create opposing forces at opposite ends of the rotor. Features of a pressure regulator are integrated into the pumping element design to passively self-balance left and right flows and atrial pressures.

The nominal external dimensions of the latest CFTAH design used in these studies are 98.4 $\mathrm{mm}$ (length), $62 \mathrm{~mm}$ (diameter), $160 \mathrm{cc}$ (volume displacement), and $486 \mathrm{~g}$ (device weight with cable and connector off the scale) (Figure 1A and B).

\section{In Vivo Study}

The CFTAH was implanted in four Jersey calves (weight range, $77.0-93.9 \mathrm{~kg}$ ) by resecting both ventricles under general anesthesia and cardiopulmonary bypass support [7]. Post implant, animals were transferred to the chronic care unit for postoperative care under continuous hemodynamic monitoring. The systemic arterial pressure (AoP, obtained from a catheter within the carotid artery) and pulmonary arterial pressure (PAP) were continuously monitored with fluid-filled pressure lines. Instantaneous pump flow was calculated from the pump speed and motor power [5]. 
The pump speed modulation study was performed on the day of elective sacrifice in four animals that had been living with the implanted CFTAH for the planned duration of 14 (one animal), 30 (one animal), or 90 days ( 2 animals). The sinusoidal pump speed modulations were performed at $\pm 15 \%$ and $\pm 25 \%$ of mean pump speed at $80 \mathrm{bpm}$ for about $10 \mathrm{~min}$ each, similar to what we reported in acute anesthetized calves [6]. Due to voltage limitations of the current controller, the maximum pump speed was truncated at 3,600 rpm when $\pm 25 \%$ of speed modulation was used. The hemodynamics (AoP, PAP, and pump flow) were evaluated and compared to the constant speed ( $0 \%$ modulation) condition. The pulsatility index was calculated by the difference between the peak and minimum pump flow rates divided by the mean pump flow rate. To further evaluate pulsatility, the energy-equivalent pressure (EEP) and surplus hemodynamic energy (SHE) were calculated using the formulas EEP $=\mathrm{Q} \times \mathrm{AoP}$ $\mathrm{dt} / \mathrm{Q} \mathrm{dt}$ and $\mathrm{SHE}=1,332 \times(\mathrm{EEP}-$ mean $\mathrm{AoP})(\mathrm{Q}$ is the instantaneous pump flow $)$ [8].

The study was approved by Cleveland Clinic's Institutional Animal Care and Use Committee, and the animals received humane care in compliance with the "Guide for the Care and Use of Laboratory Animals" (Institute of Laboratory Animal Resources, Commission on Life Sciences, National Research Council, National Academy Press, Washington, DC 2011) and institutional policy on animal care.

\section{Results}

Figure 1C shows representative waveforms of pump speed, AoP, and pump flow with sinusoidal pump speed modulations of $0 \%, \pm 15 \%$ and $\pm 25 \%$. With $\pm 15 \%$ and $\pm 25 \%$ speed modulations, the pump flow waveform and the AoP waveform showed higher pulsatility than those with no speed modulation. The AoP and PAP pulse pressures increased to 12.0 $\pm 4.1 \mathrm{~mm} \mathrm{Hg}$ and $12.3 \pm 0.5 \mathrm{~mm} \mathrm{Hg}$ at $\pm 15 \%$ speed modulation and to $15.9 \pm 5.3 \mathrm{~mm} \mathrm{Hg}$ and $15.7 \pm 0.8 \mathrm{~mm} \mathrm{Hg}$ at $\pm 25 \%$ speed modulation, respectively (Table 1 ). The first derivative (dP/dt) of the AoP significantly increased to $121 \pm 28 \mathrm{~mm} \mathrm{Hg}$ at $25 \%$ speed modulation. The pulsatility index and SHE significantly increased to $1.05 \pm 0.14$ and $1,346 \pm 479 \mathrm{ergs} / \mathrm{cm}$ at $\pm 15 \%$ speed modulation and to $1.51 \pm 0.20$ and $3,381 \pm 1,477 \mathrm{ergs} / \mathrm{cm}$ at $\pm 25 \%$ speed modulation. There were no changes in the mean AoP, mean PAP, or mean pump flow. The calves showed no behavioral changes during the pump speed modulation study.

\section{Discussion}

In this study, we demonstrated the feasibility of generating pulsatility with pump speed modulations of the CFTAH in chronic, awake, upright calves with normal sympathetic tone, confirming the results of our earlier study, performed as an open-chest procedure in acute anesthetized calves in which the sympathetic system had been dampened [6]. The augmentation in pulsatility was low (pulse pressure of $15.9 \mathrm{~mm} \mathrm{Hg}$, pulsatility index of 1.51 , and SHE of 3,381 ergs/cm at $\pm 25 \%$ modulation) probably due to (1) low flow modulation compared with the native heart and (2) higher vascular compliance of the calves. Only sinusoidal pump speed modulations at $80 \mathrm{bpm}$ were evaluated; however, as we previously demonstrated in vitro [9], a wide variety of pulsatilty can be generated with this device, including physiologic pulsation. There was some pulsatility observed at $0 \%$ speed modulation (pulse pressure of 5.6 and $9.6 \mathrm{~mm} \mathrm{Hg}$ in AoP and PAP, respectively). In these 
chronic calves with the CFTAH, the left and right atria were preserved and beating. This atrial kick, in addition to respiratory effects, caused some pulsatility even with $0 \%$ speed modulation as shown in Figure 1C.

Our group was the first (in the 1980s) to demonstrate the feasibility of nonpulsatile circulation, reporting 3-month survival in animals with a fibrillated heart totally supported by two external centrifugal pumps [10,11]. Since then, the effects of reduced pulsatility have been a subject of debate [12-15], which has recently taken on more urgency in a new era of increased use of CF LVADs as destination therapy. A critical issue in evaluating the effects of pulsatility is lack of a chronic, implantable device that can produce all the following conditions: no pulse, various degrees of pulsatility, and "physiological pulsatility" (a pulse pressure of $\sim 40 \mathrm{~mm} \mathrm{Hg}$ with no flow during two thirds of the cardiac cycle). The CFTAH has the ability to produce totally nonpulsatile flow (constant speed without speed modulation), various degrees of pulsatility (sinusoidal speed modulation of $\pm 15 \%, \pm 25 \%$, and $\pm 35 \%$ of mean speed), and physiological pulse (with optimized speed modulation to mimic physiological pulse [9]). Therefore, this CFTAH gives us a unique opportunity to directly evaluate the effects of different pulsatility with the same device in the same animal.

A limitation of this study is the very short duration (about $10 \mathrm{~min}$ ) of each speed modulation, focusing only on the immediate hemodynamic changes. End-organ function, hemolysis, microcirculation, or histopathology were not evaluated during these hemodynamic conditions and remain to be explored. There is a potential for CFTAH speed modulation to be evaluated clinically for its impact on the severity of potential complications from chronic depulsed circulation. Speed modulation may also show an advantage in terms of biocompatibility by eliminating areas of fixed low velocity and/or high residence time within the device and its conduits by inducing time-varying pulsatile pressure and bloodvelocity profiles.

\section{Conclusions}

The feasibility of generating pressure pulsatility with pump speed modulation of the CFTAH was validated. This unique platform is suitable for evaluating the physiologic impact of pulsatility and allows determination of the best speed modulations in term of magnitude, frequency, and profiles.

\section{Acknowledgments}

This work was supported with federal funding obtained from the National Heart, Lung and Blood Institute, National Institutes of Health (Bethesda, Maryland, USA), under grant 5R01HL096619 (to LARG and KF).

\section{References}

1. Jabbar HR, Abbas A, Ahmed M, Klodell CT Jr, Chang M, Dai Y, Draganov PV. The incidence, predictors and outcomes of gastrointestinal bleeding in patients with left ventricular assist device (LVAD). Dig Dis Sci. 2015; 60:3697-706. DOI: 10.1007/s10620-015-3743-4 [PubMed: 26072320]

2. Wever-Pinzon O, Selzman CH, Drakos SG, Saidi A, Stoddard GJ, Gilbert EM, Labedi M, Reid BB, Davis ES, Kfoury AG, Li DY, Stehlik J, Bader F. Pulsatility and the risk of nonsurgical bleeding in patients supported with the continuous-flow left ventricular assist device HeartMate II. Circ Heart Fail. 2013; 6:517-26. DOI: 10.1161/CIRCHEARTFAILURE.112.000206 [PubMed: 23479562] 
3. Corbett SC, Ajdari A, Coskun AU, Nayeb-Hashemi H. Effect of pulsatile blood flow on thrombosis potential with a step wall transition. ASAIO J. 2010; 56:290-5. DOI: 10.1097/MAT. 0b013e3181db2476 [PubMed: 20508499]

4. Loutzenhiser R, Griffin KA, Bidani AK. Systolic blood pressure as the trigger for the renal myogenic response: protective or autoregulatory? Curr Opin Nephrol Hypertens. 2006; 5:41-9. Review.

5. Fukamachi K, Horvath DJ, Massiello AL, Fumoto H, Horai T, Rao S, Golding LA. An innovative, sensorless, pulsatile, continuous-flow total artificial heart: device design and initial in vitro study. J Heart Lung Transplant. 2010; 29:13-20. DOI: 10.1016/j.healun.2009.05.034 [PubMed: 19782599]

6. Fumoto H, Horvath DJ, Rao S, Massiello AL, Horai T, Takaseya T, Arakawa Y, Mielke N, Chen JF, Dessoffy R, Fukamachi K, Golding LA. In vivo acute performance of the Cleveland Clinic selfregulating, continuous-flow total artificial heart. J Heart Lung Transplant. 2010; 29:21-6. DOI: 10.1016/j.healun.2009.05.035 [PubMed: 19782590]

7. Karimov JH, Moazami N, Kobayashi M, Sale S, Such K, Byram N, Sunagawa G, Horvath D, Gao S, Kuban B, Golding LA, Fukamachi K. First report of 90-day support of 2 calves with a continuousflow total artificial heart. J Thorac Cardiovasc Surg. 2015; 150:687-693. e1. DOI: 10.1016/j.jtcvs. 2015.06.023 [PubMed: 26173607]

8. Undar A, Zapanta CM, Reibson JD, Souba M, Lukic B, Weiss WJ, Snyder AJ, Kunselman AR, Pierce WS, Rosenberg G, Myers JL. Precise quantification of pressure flow waveforms of a pulsatile ventricular assist device. ASAIO J. 2005; 51:56-9. [PubMed: 15745135]

9. Shiose A, Nowak K, Horvath DJ, Massiello AL, Golding LA, Fukamachi K. Speed modulation of the continuous-flow total artificial heart to simulate a physiologic arterial pressure waveform. ASAIO J. 2010; 56:403-9. DOI: 10.1097/MAT.0b013e3181e650f8 [PubMed: 20616704]

10. Golding, LAR., Loop, FD., Nosé, Y. Clinical and experimental use of the centrifugal pump. In: Attar, S., editor. New Developments in Cardiac Assist Devices. New York: Praeger Special Studies, WB Saunders; 1985. p. 92-102.

11. Yada I, Golding LR, Harasaki H, Jacobs G, Koike S, Yozu R, Sato N, Fujimoto LK, Snow J, Olsen E, Murabayashi S, Venkatesen VS, Kiraly R, Nose Y. Physiopathological studies of nonpulsatile blood flow in chronic models. Trans Am Soc Artif Intern Organs. 1983; 29:520-5. [PubMed: 6673279]

12. Garatti A, Bruschi G, Colombo T, Russo C, Lanfranconi M, Milazzo F, Frigerio M, Vitali E. Clinical outcome and bridge to transplant rate of left ventricular assist device recipient patients: comparison between continuous-flow and pulsatile-flow devices. Eur J Cardiothorac Surg. 2008; 34:275-80. discussion 280. DOI: 10.1016/j.ejcts.2008.02.019 [PubMed: 18375138]

13. Hornick P, Taylor K. Pulsatile and nonpulsatile perfusion: the continuing controversy. J Cardiothorac Vasc Anesth. 1997; 11:310-5. [PubMed: 9161899]

14. Pruijsten RV, Lok SI, Kirkels HH, Klopping C, Lahpor JR, de Jonge N. Functional and haemodynamic recovery after implantation of continuous-flow left ventricular assist devices in comparison with pulsatile left ventricular assist devices in patients with end-stage heart failure. Eur J Heart Fail. 2012; 14:319-25. DOI: 10.1093/eurjhf/hfr181 [PubMed: 22294758]

15. Thalmann M, Schima H, Wieselthaler G, Wolner E. Physiology of continuous blood flow in recipients of rotary cardiac assist devices. J Heart Lung Transplant. 2005; 24:237-45. [PubMed: 15737748] 

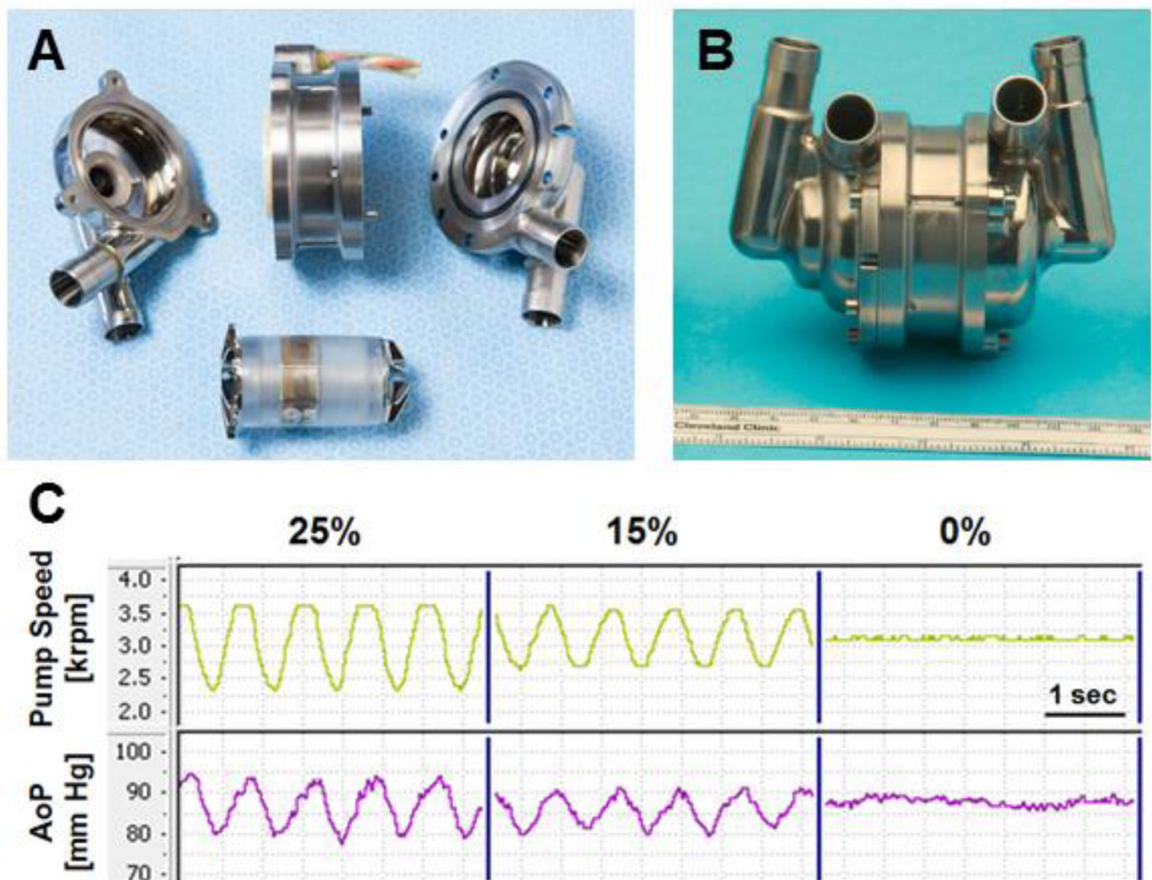

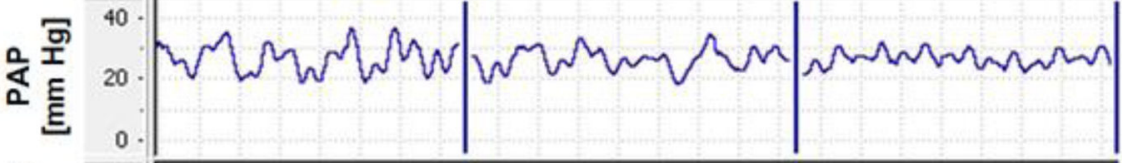

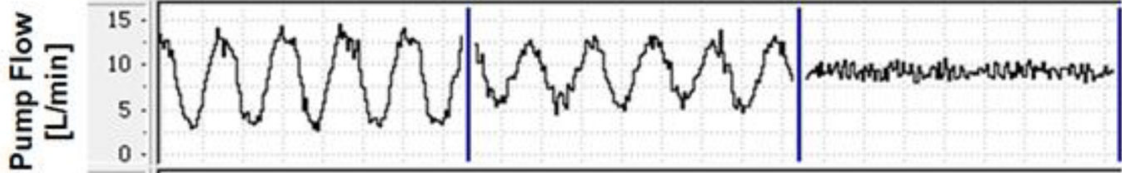

Figure 1.

A) Exploded and (B) assembled pictures of Cleveland Clinic continuous-flow total artificial heart. (C) Representative waveforms of pump speed, arterial pressure (AoP), pulmonary arterial pressure (PAP), and pump flow with pump speed modulations of $0 \%, \pm 15 \%$ and $\pm 25 \%$ 


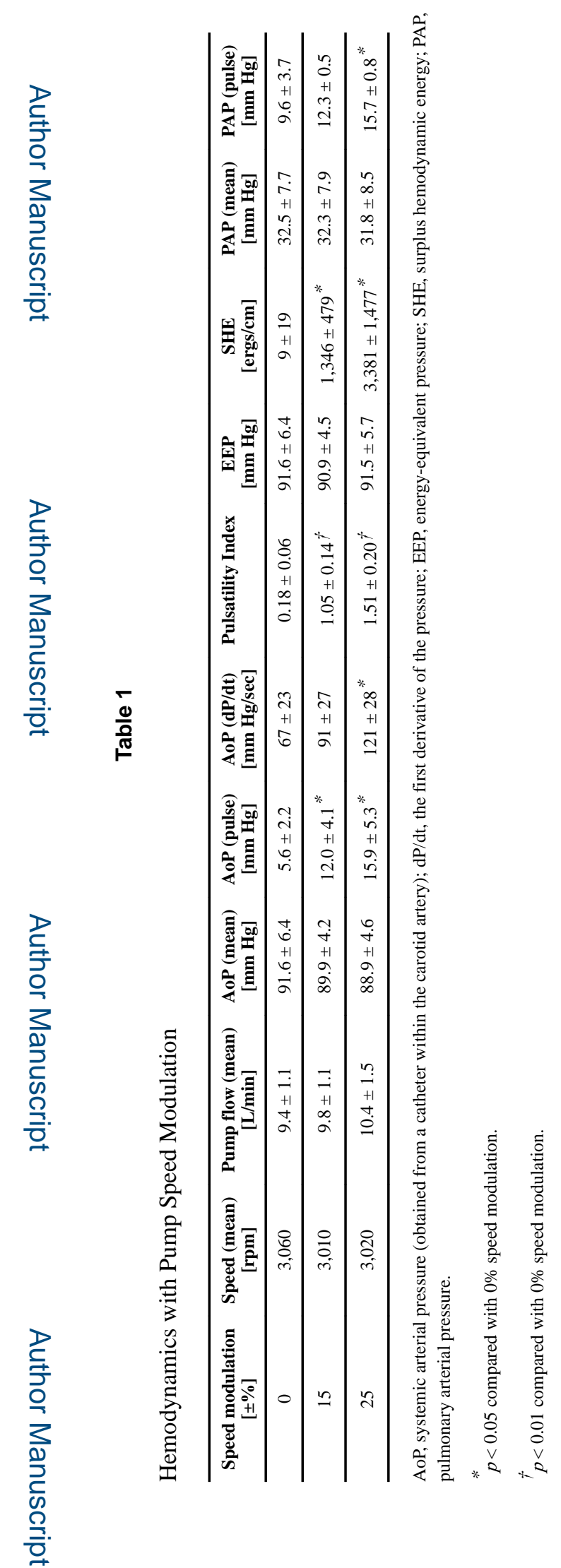

J Artif Organs. Author manuscript; available in PMC 2018 December 01. 